\title{
Reduction of the Isolated Anterior Wall of the Maxillary Sinus Fracture with Double Urinary Balloon Catheters and Fibrin Glue
}

\author{
Jaehee Kim¹, \\ Ho Jik Yang ${ }^{1}$, \\ Jong Hwan Kim ${ }^{1}$, \\ Su Jin $\mathrm{Kim}^{2}$ \\ Departments of ${ }^{1}$ Plastic and Reconstructive \\ Surgery and ${ }^{2}$ Otorhinolaryngology, Eulji \\ University College of Medicine, Daejeon, \\ Korea
}

\begin{abstract}
Background: Conservative treatment is performed for isolated anterior wall of the maxillary sinus fractures, in many cases when the fracture is clinically not severe and asymptomatic. Despite the absence of symptoms, complications such as sinusitis, rhinitis, and chronic purulent secretion may develop; therefore, successful reduction is required. We attempted to reduce the risk of complications using an alternative technique: reduction of the fracture with two urinary balloon catheters inserted through the maxillary ostium and fixation using fibrin glue, which minimizes the damage to the bony fragments and sinus mucosa.

Methods: In this study, 38 patients who were diagnosed with an isolated anterior wall of the maxillary sinus fracture at our hospital between January 2014 and January 2017 were enrolled. The fracture site was exposed via the Caldwell-Luc approach followed by reduction through the insertion of two urinary balloon catheters using a nasal endoscope and fixation with fibrin glue. The sex, cause of fracture, physical examination, and presence of complications were examined and patient's medical records and facial bone computed tomography scans were analyzed.

Results: Radiological evaluation showed that there was no evidence of collapsed reduction fragments. Although some patients had remaining symptoms of hypoesthesia (15\%; 3 patients), there were no complications such as infection, rhinitis, sinusitis, and chronic purulent secretion at the surgical site.

Conclusion: In this study, we present an alternative surgical technique using two urinary balloon catheters and fibrin glue for the successful reconstruction of an isolated anterior wall of the maxillary sinus fracture. This technique enables precise restoration with a reduced risk of complications.
\end{abstract}

Keywords: Maxillary sinus anterior wall / Fracture / Urinary balloon catheter / Fibrin glue

\section{INTRODUCTION}

Fracturing of the anterior wall of the maxillary sinus is often included in a zygomaticomaxillary complex (ZMC) fracture; isolated anterior wall of the maxillary sinus fractures account for 1.3\% of facial bone fractures [1]. In the case of an isolated fracture, conservative treatment can be considered when the degree of fracture

\section{Correspondence: Ho Jik Yang}

Department of Plastic and Reconstructive Surgery, Eulji University Hospital, Eulji University College of Medicine, 95 Dunsanseo-ro, Seo-gu, Daejeon 302-799

E-mail: drhjyang@eulji.ac.kr

Received Sep 22, 2017 / Revised Dec 5, 2017 / Accepted Dec 8, 2017 is not severe and when no symptoms are observed. Nevertheless, patients can develop symptoms of hypoesthesia caused by a fracture in the area surrounding the infraorbital foramen, and sinusitis can occur through necrotic changes of the bony fragment remaining inside the maxillary sinus in $4 \%$ of maxillary fractures; therefore, the appropriate surgical treatment is required [2].

In the surgical treatment of an isolated anterior wall of the maxillary sinus fracture, open reduction and ridged fixation is required. Large fragments can be fixed with plates and screws and small fragments can either be positioned at the bony defect or removed. Incomplete restoration of the anterior wall of the maxil- 
lary sinus can cause sinusitis, rhinitis, and chronic purulent secretion [3]. In this report, we assess the usefulness of a surgical technique that exposes the fracture site using the Caldwell-Luc approach. A urinary balloon catheter is then inserted through the maxillary ostium using an endoscope to reduce fractured segment. Fixation using fibrin glue (Tisseel, Baxter Healthcare, Norfolk, UK) is performed next to reduce the risk of damage and complications that can occur when manipulating the bony fragment during the open reduction.

\section{METHODS}

Forty-seven patients were diagnosed with an isolated anterior wall of the maxillary sinus fracture and were admitted to the Department of Plastic and Reconstructive Surgery in our hospital between January 2014 and January 2017. Among them, 38 patients were available to have their progress monitored for more than 6 months after the operation with urinary balloon catheters and fibrin glue, and were selected as study subjects, the other, 9 patients received conservative treatment as their fractures were not severe and were asymptomatic.

The patients' medical records and radiation test results were analyzed and their age, sex, cause of fracture, physical examination, and presence of complications were examined. A diagnosis was made from the medical history, physical examination, and facial bone computed tomography (CT) results.

If the patient showed symptoms of hypoesthesia or if a tiny bony fragment was observed inside the maxillary sinus, surgery was performed on average 7 days (range, 5-11 days) after the injury. The operation was performed under general anesthesia. In order to reduce intraoperative bleeding, $2 \%$ lidocaine mixed with epinephrine at a 1:100,000 ratio was injected into the intraoral upper buccal sulcus. The fractured anterior wall of the maxillary sinus was exposed via a $3 \mathrm{~cm}$ mucosal incision using the CaldwellLuc approach (Fig. 1). Using a rigid $30^{\circ}$ endoscope, the uncinate process was resected and the inner mucosa of the maxillary sinus was observed. The bloody discharge inside the sinus was aspirated using a curved aspirator to ensure the right localization. The urinary balloon catheter was inserted through the widened ostium

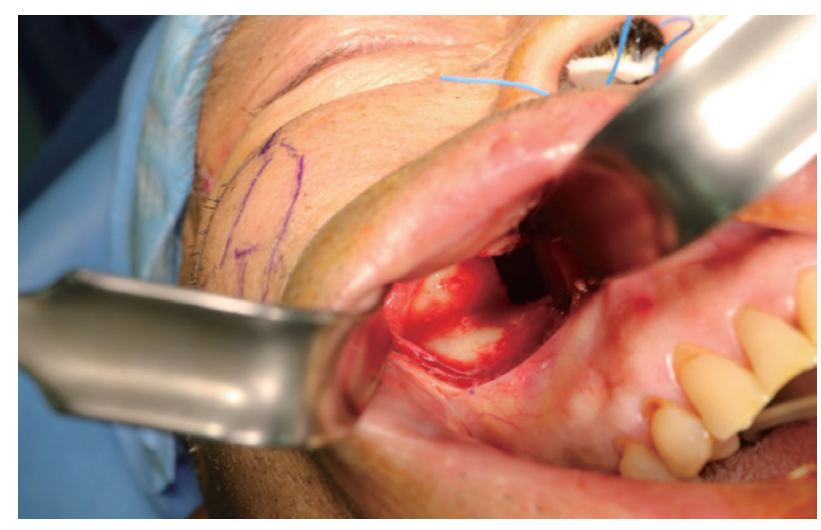

Fig. 1. The isolated anterior wall of the maxillary sinus fracture was exposed using the Caldwell-Luc approach.

into the maxillary sinus. After insertion of a 14-French urinary balloon catheter into the anterior side of the maxillary sinus and a 10-French urinary balloon catheter into the posterior side of the maxillary sinus, $12-15 \mathrm{~mL}$ and $6-10 \mathrm{~mL}$ of physiological saline solution was injected into each balloon, respectively, resulting in expansion of the balloons. After confirming proper reduction of the bony fragments through an intraoral incision (Fig. 2), the fibrin glue was sprayed into the site (Fig. 3). The intraoral incision site was repaired using Vicryl 4-0. The urinary balloon catheters were removed on postoperative day 21 .

\section{RESULTS}

The patients were aged between 18 and 63 years with a mean age of 35.6 years. Of the 38 patients, 28 were male (74\%) and 10 were female (26\%). The biggest cause of fracture was a traffic accident (17 cases), followed by violence (10 cases), slipping over ( 7 cases), sports damage (3 cases), and falling (1 case) (Table 1). The mean operation time was 92 minutes.

After the operation, the level of fracture reduction was confirmed by an immediate postoperative CT and there was no urinary balloon catheters dislocation because the diameter of sinus opening was small enough from 10-12 mm after uncinectomy. The urinary balloon catheters were removed on postoperative day 21 in all patients. A follow-up CT confirmed maintenance of the reduction state after removal of the urinary balloon catheters. 

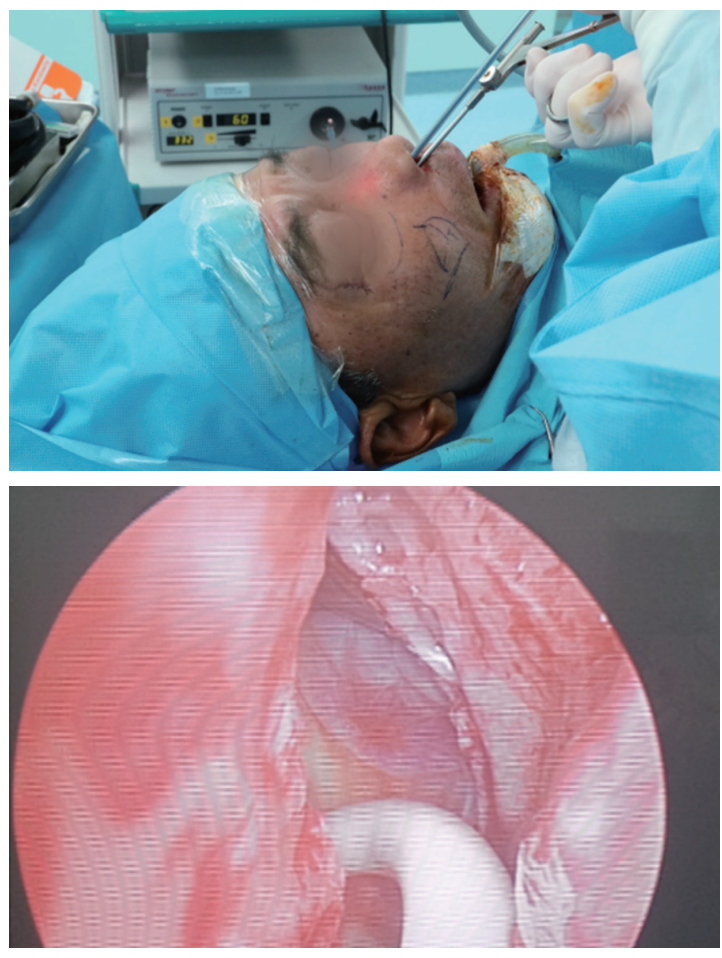

Fig. 2. Double balloon urinary catheters were inserted using a nasal endoscope.
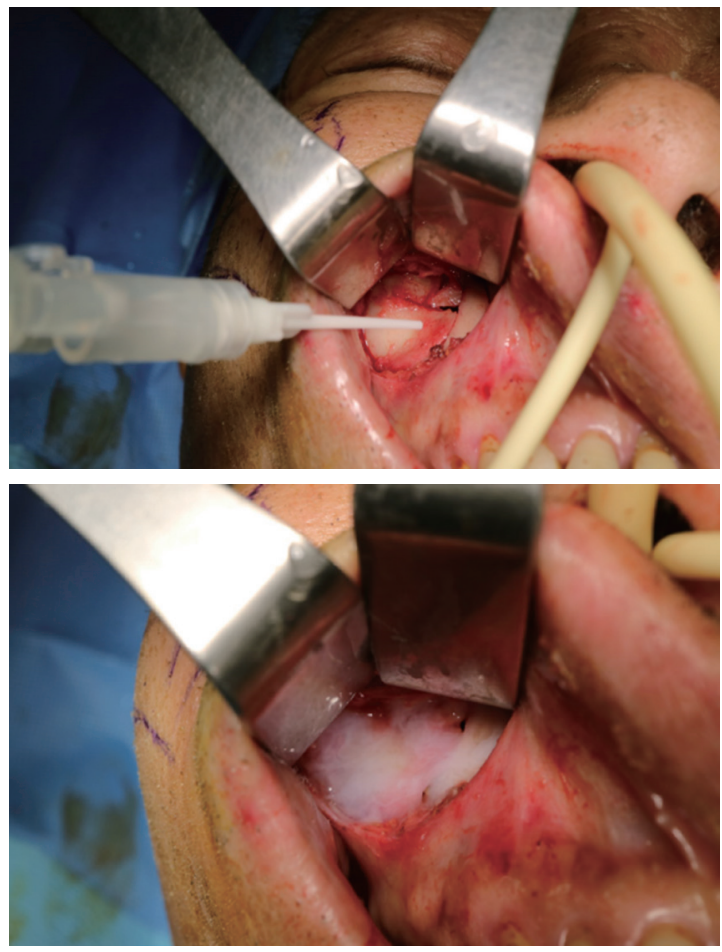

Fig. 3. Fibrin glue (Tisseel) was sprayed after the bony fragment reduction.
Table 1. Causes of isolated anterior wall of the maxillary sinus fractures

\begin{tabular}{lc} 
Cause & Patients \\
\hline Traffic accident & $17(45)$ \\
Violence & $10(26)$ \\
Slipping over & $7(18)$ \\
Sports & $3(8)$ \\
Falling & $1(3)$ \\
Total & $38(100)$ \\
\hline
\end{tabular}

Values are presented as number (\%).

Postoperative month 6 CT was performed to confirm the bone union state and the absence of sinusitis (Fig. 4).

There were no cases of postoperative infection or collapsing of the reduction fragments observed in the CT performed after removal of the urinary balloon catheters on postoperative day 21. There were 20 cases of preoperative hypoesthesia, and although the symptoms remained in 3 cases at the 6-month follow-up, there was an improvement compared to the preoperative state. There were no complications such as sinusitis, rhinitis, and chronic purulent secretion at the 6-month follow-up (Table 2).

\section{DISCUSSION}

The maxillary sinus is the largest air sinus in the human body and serves to reduce the weight of the facial skeleton and generates voice resonance [4]. As the anterior wall of the maxillary sinus is very thin and flat, fractures can easily occur by trauma such as in traffic accidents and violence. Without proper restoration of the fractured anterior wall of the maxillary sinus, sinusitis, rhinitis, prolapse of the soft tissue from the cheeks into the sinus, intrusion of bone fragments, and irritation of the mucosa can occur [5]. In the reduction of the fracture, when the size of the bony fragments are small and the number is high, fixation using plates and screws is difficult and there is a high risk of additional bony fragments being produced during the manipulation process. When the defect cannot be blocked using bony fragments, the traditional method is to restore using titanium meshes and resorbable materials; however, complications such as rhinitis, purulence, pain, and swelling have been reported when reconstruction was per- 

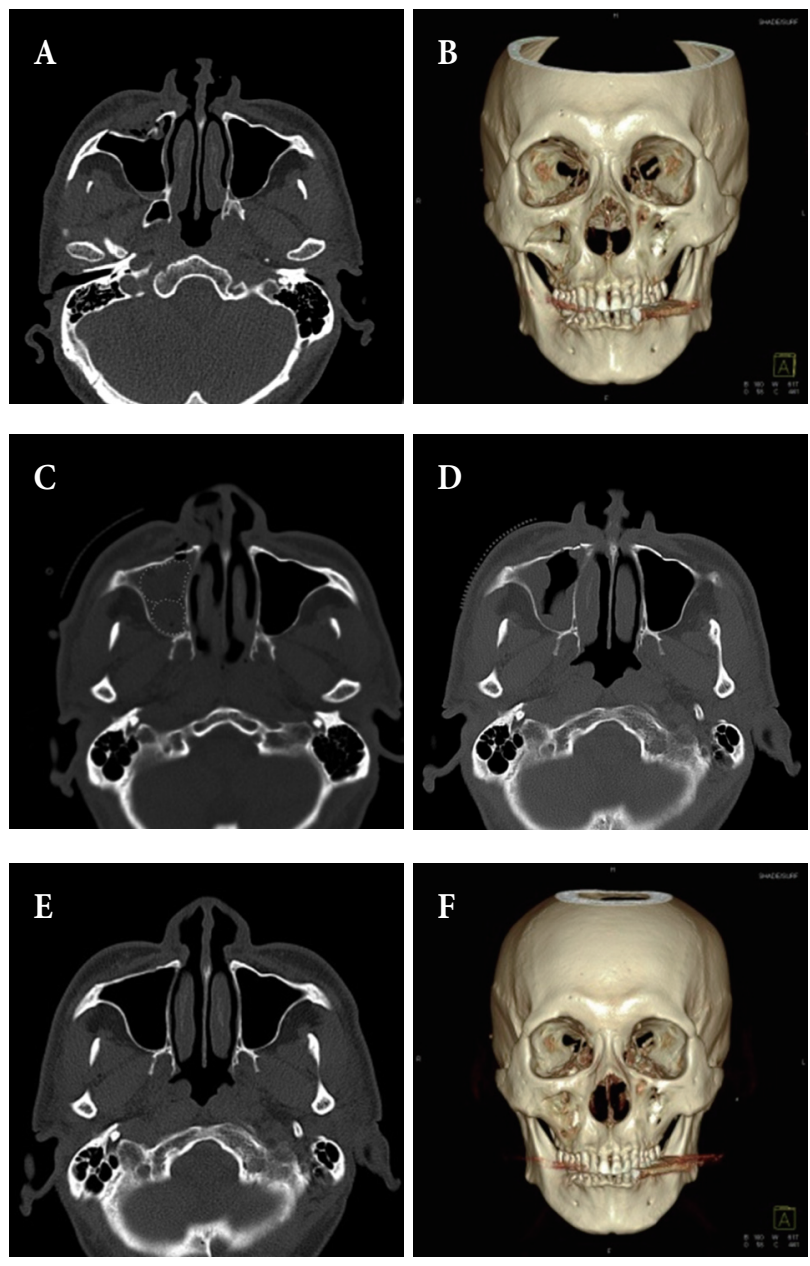

Fig. 4. A 57-year-old male patient presented with a right isolated anterior wall of the maxillary sinus fracture after falling. (A) Preoperative computed tomography (CT). (B) Preoperative CT three dimensional (3D) scan; (C) Postoperative CT. (D) Postoperative day 21 CT after removal of urinary balloon catheters. (E) Postoperative month 6 CT. (F) Postoperative month 6 CT 3D scan.

Table 2. Incidence of complication

\begin{tabular}{ll} 
Complication & Patients \\
Postoperative infection & $0 / 38(0)$ \\
Reduction fragments collapse & $0 / 38(0)$ \\
Hypoesthesia & $3 / 20(15)$ \\
Sinusitis & $0 / 38(0)$ \\
Rhinitis & $0 / 38(0)$ \\
Chronic purulent secretion & $0 / 38(0)$ \\
\hline
\end{tabular}

Values are presented as proportion (\%). formed using titanium or resorbable mesh [5].

In order to minimize the manipulation of bony fragments and damage to the mucosa, we performed reduction by insertion of urinary balloon catheters into the maxillary sinus and effectively achieved fixation using fibrin glue. The use of two urinary balloon catheters is more favorable for successful reduction of the fracture compared to using on urinary balloon catheter as it takes into account the 3-dimensional structure of the maxillary sinus. After spraying of the fibrin glue and waiting for 5 minutes for it to turn an opaque color, the open wound is closed and the operation is completed. Successful reduction was confirmed by a postoperative CT. The urinary balloon catheters were removed on postoperative day 21 and CT was conducted to examine the presence of collapsed reduction fragments.

Fibrin glue consists of a syringe containing plasmatic protein, fibrinogen, and factor XIII and a syringe containing calcium chloride, thrombin, and aprotinin. When these components are mixed, a fibrin clot is formed which acts as glue. Fibrin glue has been used in neurosurgery, breast surgery, facial lifting, and gastroenteroanastomosis; it reduces shearing force by increasing the adherence to the surrounding tissue and reduces the bleeding tendency due to a reaction similar to the last step of a physiological hemostatic reaction [6]. Initially, fibrin glue posed a risk for bloodborne diseases as it was made from donated plasma, which were small in amounts, and did not maintain sufficient quality; however, recent products such as Tisseel have been developed and are currently used in various fields [7]. The structural integrity of the fibrin clot is maintained for 3 weeks by antifibrinolytic components such as aprotinin [8]. During this time, the restoration stability is maintained not only by the fibrin glue, but also by the double urinary balloon catheters inserted into the maxillary sinus.

In this study, there were no cases of collapsing of the reduction fragments, and no complications were observed such as infection, rhinitis, sinusitis, and chronic purulent secretion at the surgical site. This is likely to be because the inside of the maxillary sinus was filled and blocked from the outside by the double balloon catheters in addition to the healing of the soft tissues and regeneration of the sinus mucosa while the fibrin clot is maintained. On 
the other hand, Thompson and Davis [9] indicated the increased risk of infection with the use of fibrin glue, and Tanemoto and Fujinami [10] suggested a risk of bacterial colonization. Therefore, when conducting surgery on a highly contaminated wound, use of urinary balloon catheters and fibrin glue needs to be carefully considered.

The surgical technique for an isolated anterior wall of the maxillary sinus fracture involving reduction using urinary balloon catheters and fixation using fibrin glue is advantageous as it allows precise restoration and reduces the risk of complications when compared to surgical techniques involving direct manipulation of the bony fragments and fixation using plates and screws, or the use of titanium mesh. Therefore, it should be considered as a surgical method for reconstruction of this fracture.

\section{CONFLICT OF INTEREST}

No potential conflict of interest relevant to this article was reported.

\section{PATIENT CONSENT}

The patient provided written informed consent for the publica- tion and the use of their images.

\section{REFERENCES}

1. Hwang K, You SH. Analysis of facial bone fractures: an 11-year study of 2,094 patients. Indian J Plast Surg 2010;43:42-8.

2. Perko M. Maxillary sinus and surgical movement of maxilla. Int J Oral Surg 1972;1:177-84.

3. Blondel JH, Legros M. Post-traumatic maxillary sinusitis. J Fr Otorhinolaryngol Audiophonol Chir Maxillofac 1980;29:633-4.

4. Chanavaz M. Maxillary sinus: anatomy, physiology, surgery, and bone grafting related to implantology: eleven years of surgical experience (1979-1990). J Oral Implantol 1990;16:199-209.

5. Ballon A, Landes CA, Zeilhofer HF, Herzog M, Klein C, Sader R. The importance of the primary reconstruction of the traumatized anterior maxillary sinus wall. J Craniofac Surg 2008;19:505-9.

6. Grossman JA, Capraro PA. Long-term experience with the use of fibrin sealant in aesthetic surgery. Aesthet Surg J 2007;27:558-62.

7. Gibble JW, Ness PM. Fibrin glue: the perfect operative sealant? Transfusion 1990;30:741-7.

8. Radosevich M, Goubran HI, Burnouf T. Fibrin sealant: scientific rationale, production methods, properties, and current clinical use. Vox Sang 1997;72:133-43.

9. Thompson DF, Davis TW. The addition of antibiotics to fibrin glue. South Med J 1997;90:681-4.

10. Tanemoto K, Fujinami H. Experimental study on bacterial colonization of fibrin glue and its prevention. Clin Ther 1994;16:1016-27. 